\title{
Asociación entre el espesor de la grasa subcutánea abdominal y la esteatosis hepática, las enzimas hepáticas y los lípidos séricos en niños obesos Association of abdominal subcutaneous fat thickness with hepatic
steatosis, liver enzymes, and serum lipids in obese children
}

\author{
Dr. Levent Soydan ${ }^{a}$, Dra. Narin Akıcı y Dra. Yesim Coskun
}

\section{RESUMEN}

Introducción. La obesidad infantil puede causar hiperlipidemia y esteatosis hepática y complicaciones crónicas. Nuestro objetivo fue evaluar la relación entre el espesor de la grasa subcutánea abdominal (GSA) y la esteatosis hepática, las transaminasas y los lípidos séricos en niños obesos.

Población y métodos. Estudio retrospectivo en niños (4-18 años) que acudieron a los consultorios externos. Se evaluaron las asociaciones entre el espesor de la GSA mediante ecografía y la esteatosis hepática, alanina aminotransferasa (ALT), aspartato aminotransferasa (AST) y el perfil lipídico sérico. Resultados. Se identificaron 95 niños con esteatosis hepática; el grado 1 fue el más frecuente $(73,6 \%$, n: 70$)$, seguido del grado 2 $(21,1 \%)$ y del $3(5,3 \%)$. El espesor medio (en $\mathrm{mm}$ ) de la GSA de línea media y flancos fue $38,48 \pm 11,53$ y $20,91 \pm 8,00$ en grado $1 ; 41,23 \pm 10,03$ y $19,84 \pm 6,75$ en grado 2, y $63,12 \pm 12,08$ y $23,22 \pm 5,92$ en grado 3 , respectivamente. El espesor de la GSA de línea media y flancos se correlacionó positivamente con triglicéridos en grado 3; el espesor de la GSA de línea media se correlacionó con el índice de masa corporal, colesterol total, colesterol LDL y AST (r: 0,9; p: 0,037; r: 0,648; p: 0,001; r: 0,387; p: 0,001; r:0,406; $p: 0,001 ; \mathrm{r}: 0,463 ; p: 0,001$; respectivamente) en grado 1 .

Conclusión. El espesor de la GSA mediante ecografía puede predecir hiperlipidemia en niños obesos con esteatosis hepática de grado 3 e hipercolesterolemia en grado 1.

Palabras clave: grasa subcutánea abdominal, obesidad infantil, esteatosis hepática, metabolismo lipídico.

http:/ / dx.doi.org/10.5546/aap.2021.114

Texto completo en inglés:

http:/ / dx.doi.org/10.5546/ aap.2021.eng.114

Dr. Levent Soydan:: levent.soydan@gmail. com

Financiamiento:

Ninguno.

Conflicto de intereses:

Ninguno que declarar.

Cómo citar: Soydan L, Akıcı N, Coskun Y. Asociación entre el espesor de la grasa subcutánea abdominal y la esteatosis hepática, las enzimas hepáticas y los lípidos séricos en niños obesos. Arch Argent Pediatr 2021;119(2):114-122.

\section{GLOSARIO}

ALT: alanina aminotransferasa.

AST: aspartato aminotransferasa.

C-HDL: colesterol de las

lipoproteínas de alta densidad.

C-LDL: colesterol de las

lipoproteínas de baja densidad.

CT: colesterol total.

EHNA: esteatosis hepática no

alcohólica.

GSA: grasa subcutánea abdominal.

IMC: índice de masa corporal.

RM: resonancia magnética.

TC: tomografía computada.

TG: triglicéridos.

\section{INTRODUCCIÓN}

La obesidad infantil es un importante problema de salud que se caracteriza por un exceso de adiposidad o grasa corporal. Es uno de los factores responsables de problemas médicos, físicos y emocionales durante la niñez que pueden ocasionar una elevada morbilidad en la adultez. Según la Organización Mundial de la Salud, la prevalencia de la obesidad aumentó en las últimas tres décadas con un incremento anual del $0,5 \%$ al $1 \%{ }^{2,3}$ La obesidad es una enfermedad multifactorial que se ve afectada por factores genéticos y no genéticos. ${ }^{1,2} \mathrm{Se}$ define en función del índice de masa corporal (IMC), que tiene una fuerte correlación con la adiposidad. ${ }^{4}$

La obesidad infantil frecuentemente persiste en la edad adulta, y esto se relaciona en gran medida con la resistencia a la insulina, la diabetes de tipo 2, la hipertensión, la dislipidemia, la esteatosis hepática, 
el asma, la apnea del sueño y los problemas ortopédicos..$^{5-8}$ La obesidad puede asociarse a alteraciones bioquímicas que se inician durante la niñez. El perfil lipídico sérico, en especial la concentración de triglicéridos y de colesterol total (CT) y la concentración sérica de alanina/ aspartato aminotransferasa (ALT/AST), puede estar por encima del intervalo normal en los niños obesos. ${ }^{9,10}$ La obesidad infantil también puede asociarse al esteatosis hepática, que se define como la acumulación de grasa en el hígado, especialmente en forma de triglicéridos. Si bien la esteatosis hepática suele ser una enfermedad autolimitada, puede evolucionar a esteatosis hepática no alcohólica (EHNA) y cirrosis hepática. ${ }^{11,12}$

La cantidad y la distribución del tejido adiposo en el cuerpo están estrechamente asociadas a la obesidad; se realizaron varios intentos para evaluar el tejido adiposo visceral y subcutáneo mediante ecografía, radioabsorciometría de doble energía, tomografía computada (TC) y resonancia magnética (RM). ${ }^{13}$ Con el objetivo de estudiar la asociación entre la adiposidad corporal y los factores de riesgo relacionados con la obesidad, varios estudios se enfocaron particularmente en las medidas antropométricas y la adiposidad visceral. Aún no se ha establecido claramente la capacidad que tiene el espesor de la grasa subcutánea abdominal (GSA) como parámetro único para reflejar el grado de esteatosis hepática e hiperlipidemia. La ecografía puede utilizarse para diagnosticar la esteatosis hepática y para medir el espesor de la GSA. ${ }^{14-16}$ Si el espesor de la GSA permite predecir estos parámetros relacionados con la obesidad en niños obesos, entonces es posible agregar la evaluación ecográfica del espesor de la GSA a los estudios diagnósticos iniciales de los niños obesos, ya que la ecografía es un método de diagnóstico por imágenes seguro, no invasivo, fácil de usar y ampliamente disponible. ${ }^{13}$

El objetivo de este estudio fue evaluar la asociación entre el espesor de la GSA y la esteatosis hepática, el perfil lipídico sérico y la concentración de transaminasas en niños obesos.

\section{POBLACIÓN Y MÉTODOS}

Este estudio retrospectivo se realizó en niños que acudieron a los consultorios pediátricos externos por obesidad entre mayo de 2016 y abril de 2018, en el Hospital de Formación e Investigación Haydarpaşa Numune. Se definió que un paciente pediátrico era obeso si su IMC se encontraba en el percentilo 95 o más. ${ }^{17} \mathrm{Se}$ excluyó a los niños obesos con hepatopatías crónicas, hepatopatías infecciosas, hepatomegalia, dislipidemias congénitas o metabolopatías.

Los datos relativos a la estatura y el peso de los niños se habían tomado de forma inmediata en el ingreso. Luego se combinaron estos datos con la edad y el sexo de los niños para calcular el IMC mediante la fórmula matemática del índice de peso por estatura. La concentración de

Figura 1. Medición del espesor de la grasa subcutánea abdominal de los flancos desde la superficie de la piel (entre los cursores)

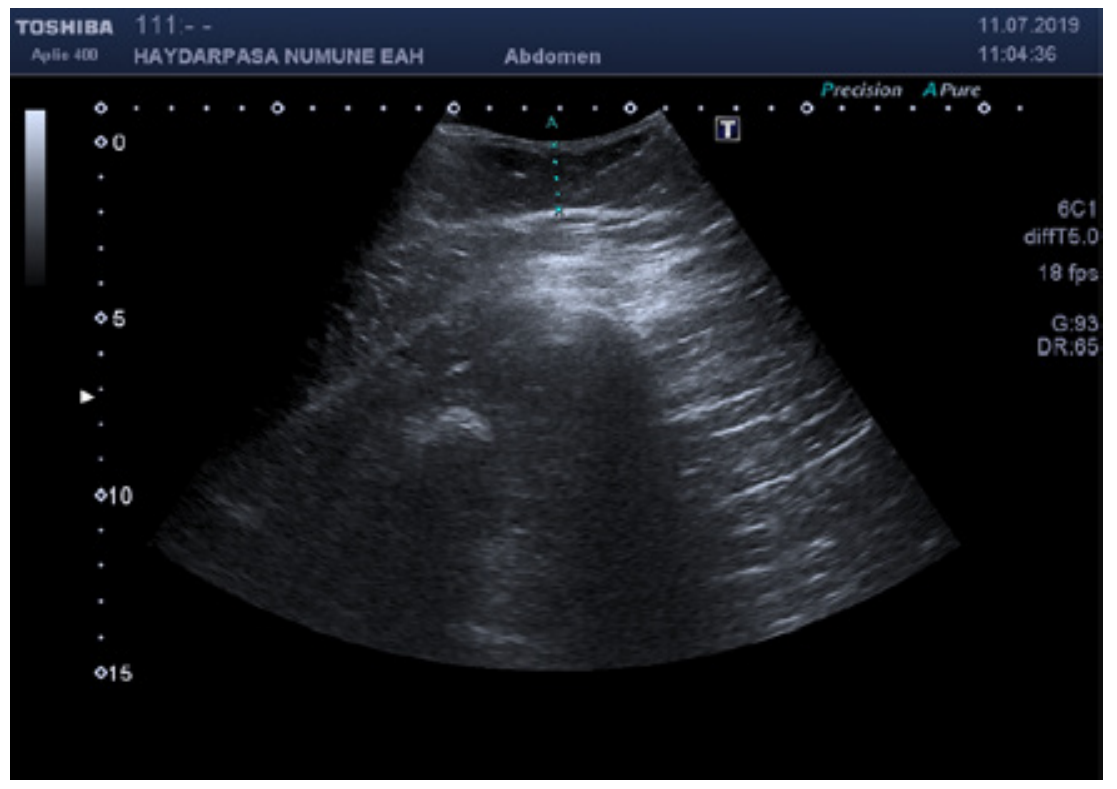


transaminasas y lípidos séricos (CT, colesterol de las lipoproteínas de baja densidad [C-LDL], colesterol de las lipoproteínas de alta densidad [C-HDL], triglicéridos [TG]) se obtuvieron en ayunas mediante venopunción en el transcurso de los 7 días posteriores al ingreso en los consultorios externos.

Un único radiólogo, que tenía información sobre la presentación clínica del paciente, pero desconocía los datos de laboratorio, realizó una ecografía convencional. El procedimiento se llevó a cabo con un ecógrafo Aplio 500 (Toshiba/ Canon, Japón) con transductores lineales y convexos (ancho de banda de frecuencia: $18 \mathrm{MHz}$ y 3,5-6 MHz, respectivamente) de forma estandarizada: el espesor de la GSA se midió aplicando una presión mínima con el transductor mientras el paciente respiraba normalmente en decúbito supino. El espesor de la GSA de la línea media se midió en el plano transversal, un centímetro por debajo del ombligo, y el espesor de la GSA de los flancos se midió dentro del mismo plano transversal, tanto en el flanco izquierdo como el derecho, aproximadamente al nivel de ambas espinas ilíacas anterosuperiores. El valor medio entre los dos lados se utilizó para el espesor de la GSA de los flancos. El espesor de la grasa subcutánea se definió como la distancia entre la superficie de la piel y el borde externo de la capa muscular del abdomen (Figuras 1 y 2).

El grado de esteatosis hepática se determinó mediante ecografía convencional de la siguiente manera: se definió como grado 1 un aumento leve y difuso de los ecos finos del parénquima con una visualización normal del diafragma y de los bordes de la vena porta; se definió como grado 2 un aumento moderado y difuso de los ecos finos con una visualización ligeramente deficiente de los bordes de la vena porta y del diafragma; y se definió como grado 3 la presencia de ecos finos con mala visualización o falta de visualización de los bordes de la vena porta, del diafragma y del segmento posterior del lóbulo derecho. ${ }^{18}$

Consideraciones éticas: el protocolo del estudio fue aprobado por el Comité de Ética del Hospital de Formación e Investigación en Enfermedades Materno Infantiles Zeynep Kamil (aprobación $n^{\circ}$ 2018-84). El estudio se llevó a cabo conforme a los principios establecidos en la Declaración de Helsinki.

Análisis estadístico: el análisis estadístico se realizó con el programa NCSS (Number Cruncher Statistical System) 2007 (Kaysville, Utah, EE. UU.). Los datos descriptivos se expresaron como número y porcentaje o media, desviación estándar, mediana, intervalo y puntaje $Z$, según correspondiera. Se utilizaron la prueba de Kruskal-Wallis y la prueba de la U de MannWhitney con la corrección de Bonferroni para comparar tres grupos o más con distribución anormal. Se utilizó la prueba de Fisher-FreemanHalton para comparar las variables categóricas. La correlación entre las variables paramétricas se evaluó con la prueba de correlación de Spearman.

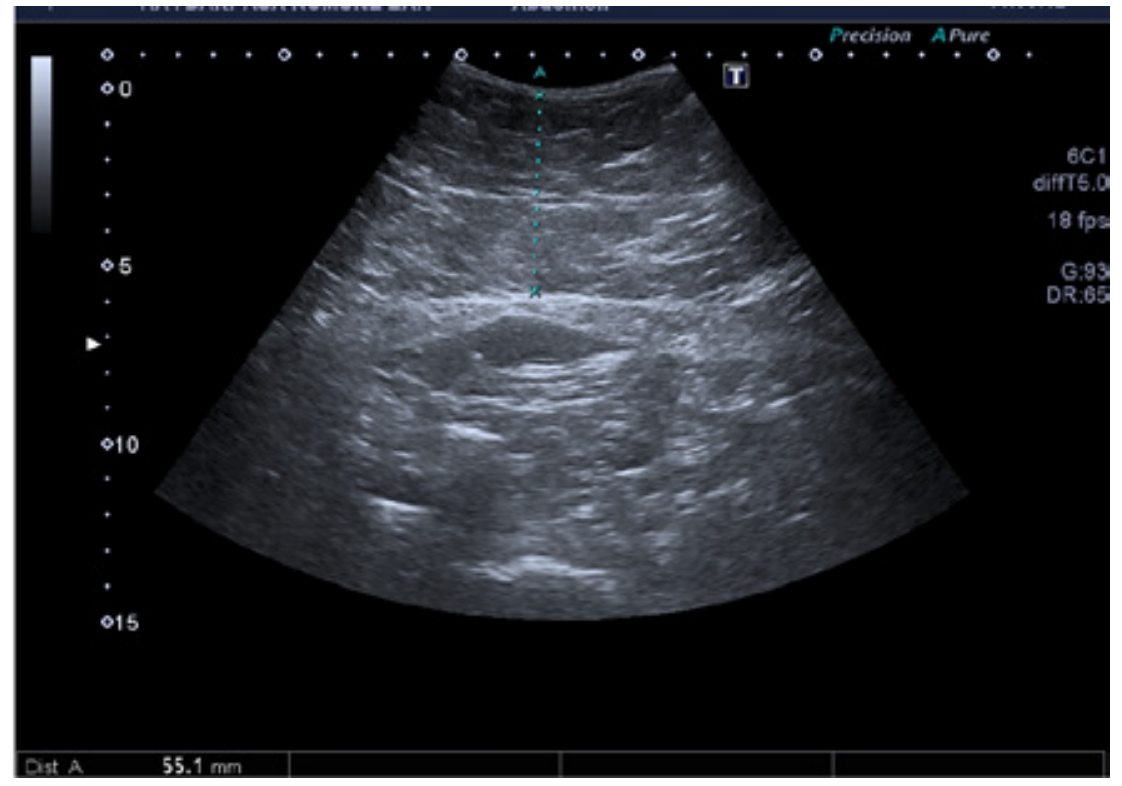


TABLA 1. Características demográficas y clínicas de los pacientes

\begin{tabular}{lll}
\hline Edad (años) & Mín.-máx. (mediana) & $4-18(11)$ \\
& Media \pm desviación estándar & $11,8 \pm 3,9$ \\
Sexo, $\mathbf{n}(\%)$ & Femenino & $40(42,1)$ \\
& Masculino & $55(57,9)$ \\
IMC (kg/m²) & Media & 28,02 \\
& Puntaje Z medio & $-1,7$ \\
TG (mg/dl) & Mín.-máx. (mediana) & $35-292(93)$ \\
& Media \pm desviación estándar & $102,03 \pm 44,43$ \\
CT (mg/dl) & Mín.-máx. (mediana) & $74-279(166)$ \\
& Media \pm desviación estándar & $166,41 \pm 37,59$ \\
C-HDL (mg/dl) & Mín.-máx. (mediana) & $23-144(45)$ \\
& Media \pm desviación estándar & $47,78 \pm 18,38$ \\
C-LDL (mg/dl) & Mín.-máx. (mediana) & $32-154(105)$ \\
& Media \pm desviación estándar & $101,31 \pm 27,85$ \\
AST (UI/l) & Mín.-máx. (mediana) & $11-94(22)$ \\
& Media \pm desviación estándar & $24,72 \pm 11,84$ \\
ALT (UI/l) & Mín.-máx. (mediana) & $9-109(21)$ \\
Espesor de la GSA de la línea media (cm) & Media \pm desviación estándar & $26,22 \pm 17,13$ \\
& Mín.-máx. (mediana) & $16-80(40,60)$ \\
Espesor de la GSA de los flancos (cm) & Media \pm desviación estándar & $40,36 \pm 12,43$ \\
Esteatosis hepática, $\mathbf{n}$ (\%) & Mín.-máx. (mediana) & $7-44,4(20,50)$ \\
& Media \pm desviación estándar & $20,80 \pm 7,63$
\end{tabular}

IMC: índice de masa corporal; TG: triglicéridos; CT: colesterol total; C-HDL: colesterol de las lipoproteínas de alta densidad; C-LDL: colesterol de las lipoproteínas de baja densidad; AST: aspartato aminotransferasa; ALT: alanina aminotransferasa; GSA: grasa subcutánea abdominal.

TABLA 2. Características demográficas y clínicas de los grupos según el grado de esteatosis hepática

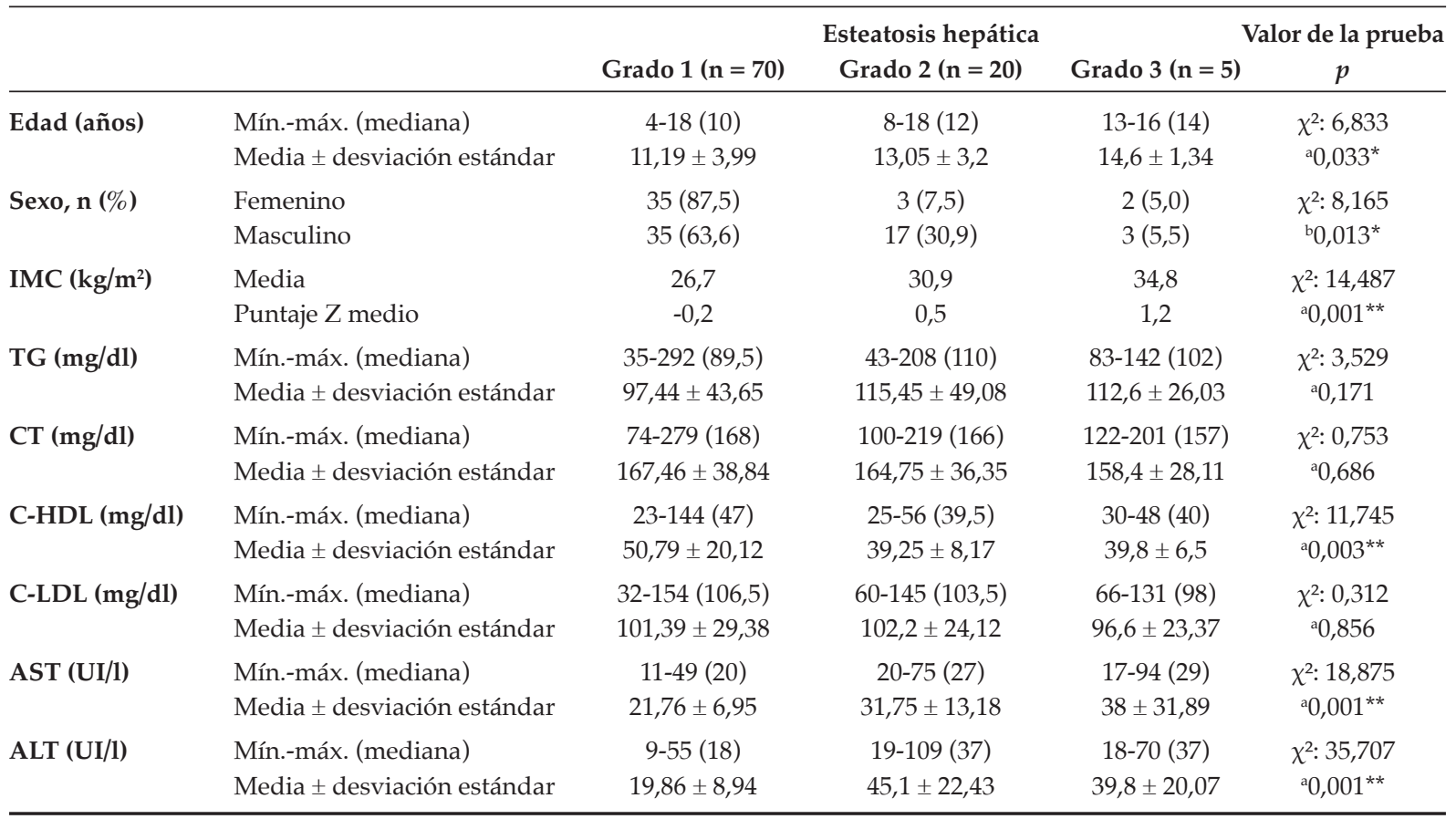

${ }^{a}$ Prueba de Kruskal-Wallis. ${ }^{\mathrm{b}}$ Fisher-Freeman. ${ }^{*} \mathrm{p}<0,05 .{ }^{* *} \mathrm{p}<0,01$.

IMC: índice de masa corporal; TG: triglicéridos; CT: colesterol total; C-HDL: colesterol de las lipoproteínas de alta densidad; C-LDL: colesterol de las lipoproteínas de baja densidad; AST: aspartato aminotransferasa; ALT: alanina aminotransferasa. 
Se estableció un error de tipo I del $5 \%$ global para inferir la significación estadística.

\section{RESULTADOS}

Se identificó a 95 niños obesos (varones: $57,9 \%$ ); la edad media era 11,8 años $\pm 3,9$ años (intervalo: 4 años-18 años). El IMC medio era de 28,0 con un puntaje $Z$ medio de $-1,7 \mathrm{~kg} / \mathrm{m}^{2}$ (Tabla 1).

Se detectó esteatosis hepática en todos los niños, y el tipo más frecuente fue el grado 1 $(73,6 \%, \mathrm{n}: 70)$, seguido del grado $2(21,1 \%$, n: 20$)$ y del grado $3(5,3 \%, n: 5)$. La edad media de los pacientes con esteatosis de grado 2 fue significativamente mayor que la de aquellos que tenían grado $1(p: 0,041)$. La esteatosis de grados 1 y 2 fue más frecuente en los varones que en las mujeres ( $p$ : 0,027 y p: 0,018, respectivamente). Los pacientes con esteatosis de grado 2 tenían un IMC significativamente mayor que aquellos con esteatosis de grado 1 ( $p: 0,001)$. Las concentraciones medias de AST y ALT fueron menores en los pacientes con esteatosis de grado 1 en comparación con aquellos que tenían esteatosis de grado 2 ( $p$ : 0,0035 y $p<0,0001$ ). Si bien las concentraciones de CT y TG no difirieron según los grados de esteatosis, los pacientes con esteatosis de grado 1 tenían una concentración media de C-HDL mayor que aquellos con grado 2 ( $p: 0,0003)$ y grado 3 ( $p: 0,0141)$.

Se observó un mayor espesor de la GSA de la línea media en los pacientes con esteatosis de grado 3 en comparación con aquellos con esteatosis de grado 1 ( $p: 0,0086)$ o grado 2

TABLA 3. Evaluación del espesor de la GSA de la línea media y de los flancos según el grado de esteatosis hepática

\begin{tabular}{llcccc}
\hline & & \multicolumn{3}{c}{ Esteatosis } & \multicolumn{3}{c}{ Valor de la prueba } \\
& & Grado 1 $(\mathbf{n}=\mathbf{7 0})$ & Grado 2 (n= 20) & Grado 3 (n= 5) & $p$ \\
\hline Espesor de la GSA de & Mín.-máx. (mediana) & $16-61(38,5)$ & $25-57(42,5)$ & $46,6-80(64)$ & $\chi^{2: 11,762}$ \\
la línea media (mm) & Media \pm desviación estándar & $38,48 \pm 11,53$ & $41,23 \pm 10,03$ & $63,12 \pm 12,08$ & ${ }^{a} 0,003^{* *}$ \\
Espesor de la GSA & Mín.-máx. (mediana) & $7-44,4(20,5)$ & $11-37,4(18,95)$ & $17-33(22)$ & $\chi^{2: 1,449}$ \\
de los flancos (mm) & Media \pm desviación estándar & $20,91 \pm 8,00$ & $19,84 \pm 6,75$ & $23,22 \pm 5,92$ & ${ }^{a} 0,485$ \\
\hline
\end{tabular}

a Prueba de Kruskal-Wallis. ${ }^{* *} \mathrm{p}<0,01$.

GSA: grasa subcutánea abdominal.

TABLa 4. Asociación entre el espesor de la GSA de la línea media y de los flancos y el IMC, los TG, el CT, el C-HDL, el C-LDL, la AST y la ALT según el grado de esteatosis hepática

\begin{tabular}{|c|c|c|c|c|c|c|c|}
\hline & & \multicolumn{3}{|c|}{$\begin{array}{l}\text { Espesor de la GSA } \\
\text { de la línea media } \\
\text { Esteatosis hepática }\end{array}$} & \multicolumn{3}{|c|}{$\begin{array}{l}\text { Espesor de la GSA } \\
\text { de los flancos } \\
\text { Esteatosis hepática }\end{array}$} \\
\hline & & Grado 1 & Grado 2 & Grado 3 & Grado 1 & Grado 2 & Grado 3 \\
\hline \multirow[t]{2}{*}{ IMC } & $\mathbf{r}$ & 0,648 & 0,558 & 0,000 & 0,233 & 0,149 & 0,300 \\
\hline & $p$ & $0,001^{* *}$ & $0,011^{*}$ & 1,000 & 0,052 & 0,531 & 0,624 \\
\hline \multirow[t]{2}{*}{ TG } & $\mathbf{r}$ & 0,009 & 0,148 & 0,900 & 0,154 & 0,048 & 0,900 \\
\hline & $p$ & 0,938 & 0,534 & $0,037^{*}$ & 0,202 & 0,840 & $0,037^{*}$ \\
\hline \multirow[t]{2}{*}{ CT } & $\mathbf{r}$ & 0,387 & 0,041 & 0,700 & 0,089 & 0,080 & 0,800 \\
\hline & $p$ & $0,001^{* *}$ & 0,862 & 0,188 & 0,464 & 0,738 & 0,104 \\
\hline \multirow[t]{2}{*}{ C-HDL } & $\mathbf{r}$ & 0,216 & 0,412 & 0,200 & 0,198 & 0,067 & 0,300 \\
\hline & $p$ & 0,072 & 0,071 & 0,747 & 0,101 & 0,780 & 0,624 \\
\hline \multirow[t]{2}{*}{ C-LDL } & $\mathbf{r}$ & 0,406 & 0,375 & 1,000 & 0,099 & 0,025 & 0,700 \\
\hline & $p$ & $0,001^{* *}$ & 0,104 & $0,001^{* *}$ & 0,415 & 0,917 & 0,188 \\
\hline \multirow[t]{2}{*}{ AST } & $\mathbf{r}$ & 0,463 & 0,133 & 0,500 & 0,431 & 0,126 & 0,900 \\
\hline & $p$ & $0,001^{* *}$ & 0,575 & 0,391 & $0,001^{* *}$ & 0,598 & $0,037^{*}$ \\
\hline \multirow[t]{2}{*}{ ALT } & $\mathbf{r}$ & 0,080 & 0,232 & 0,700 & 0,039 & 0,047 & 0,800 \\
\hline & $p$ & 0,513 & 0,325 & 0,188 & 0,750 & 0,845 & 0,104 \\
\hline
\end{tabular}

r: coeficiente de correlación de Spearman. * $\mathrm{p}<0,05 .{ }^{* *} \mathrm{p}<0,01$.

GSA: grasa subcutánea abdominal; IMC: índice de masa corporal; TG: triglicéridos; CT: colesterol total;

C-HDL: colesterol de las lipoproteínas de alta densidad; C-LDL: colesterol de las lipoproteínas de baja densidad;

AST: aspartato aminotransferasa; ALT: alanina aminotransferasa. 
( $p:$ 0,0114). El espesor de la GSA de los flancos fue similar entre los diferentes grados de esteatosis hepática (Tabla 3).

También se observó una correlación positiva entre el IMC y el espesor de la GSA de la línea media en los pacientes con esteatosis de grado 1 (r: 0,$648 ; p: 0,001)$ y grado 2 (r: 0,558 ; $p: 0,011$ ). Respecto a las transaminasas, la AST se correlacionó con el espesor de la GSA de la línea media y de los flancos en la esteatosis hepática de grado 1 (r: 0,463; p: 0,001; y r: 0,431; p: 0,001; respectivamente) y con el espesor de la GSA de los flancos en la de grado 3 ( $r: 0,900 ; p$ : 0,037). También se observó una correlación positiva entre el espesor de la GSA de la línea media y las concentraciones de C-LDL y CT en los pacientes con esteatosis de grado 1 (r: 0,406; $p$ : 0,001; y r: 0,387; $p$ : 0,001; respectivamente). En los pacientes con esteatosis de grado 3, se halló una correlación positiva entre el espesor de la GSA de la línea media y las concentraciones de TG (r: 0,900; $p: 0,037)$ y de C-LDL (r: 1,000; $p: 0,001$ ). El espesor de la GSA de los flancos también tuvo una correlación positiva con la concentración de TG en los pacientes con esteatosis de grado 3 (r: 0,900; p: 0,037; Tabla 4).

\section{DISCUSIÓN}

La obesidad infantil es un factor de riesgo potencial de enfermedades cardiovasculares como la hipertensión, la dislipidemia, la diabetes o la apnea del sueño en la adultez. Por lo tanto, es importante identificar cualquier afección relacionada con la obesidad durante la niñez que pueda empeorar o generar complicaciones cardiometabólicas posteriormente. ${ }^{1,2,19,20}$ En este estudio, se proporciona información importante sobre la asociación entre el espesor de la GSA y varios biomarcadores de dichas enfermedades relacionadas con la obesidad, los cuales podrían utilizarse para predecir el riesgo de complicaciones en niños obesos. De conformidad con esto, nuestros resultados mostraron que el espesor de la GSA podría tener un valor predictivo para la dislipidemia en pacientes pediátricos obesos, particularmente en aquellos con esteatosis hepática de grado 1.

La esteatosis hepática es una de las hepatopatías crónicas más frecuentes en la niñez $\mathrm{y}$, al igual que en los adultos, es un rasgo característico de la EHNA. ${ }^{21,22}$ La obesidad infantil se asocia a un mayor riesgo de EHNA, con hasta un $38 \%$ de prevalencia en los adolescentes..$^{21} \mathrm{La}$ EHNA incluye esteatosis hepática, que abarca de la esteatosis leve a la esteatohepatitis y, en casos aislados, la cirrosis con tendencia a un empeoramiento. ${ }^{23,24}$ En nuestro estudio, se observó que la edad media para la esteatosis hepática de grado 3 era de 14 años, mientras que para la esteatosis de grado 1 era de 10 años. Estos hallazgos sugieren que un diagnóstico y un tratamiento tempranos del esteatosis hepática pueden resultar fundamentales debido a su tendencia a agravarse. Según lo informado, la esteatosis hepática es más frecuente entre los varones, con una proporción entre varones y mujeres de 2:1. ${ }^{24}$ En nuestro estudio, también se observó que la cantidad de varones con esteatosis de grado 1 y 2 fue superior que en las mujeres y que el sexo masculino conllevaba un mayor riesgo de esteatosis hepática que el femenino. Si bien se sabe que la obesidad atenúa las diferencias en función del sexo en la esteatosis visceral, se informó que los varones puberales y pospuberales eran más propensos a tener más tejido adiposo en la región abdominal. ${ }^{25}$ Teniendo en cuenta la edad media de nuestra población, nuestros hallazgos parecen coincidir con la bibliografía, aunque es preciso esclarecerlos mejor mediante estudios bien diseñados. También se sabe que entre el $20 \%$ y el $80 \%$ de los niños obesos con esteatosis hepática pueden tener hipertrigliceridemia, C-LDL alto y concentraciones elevadas de transaminasas. ${ }^{23,24}$

Además de las mediciones antropométricas más comunes, como el grosor de los pliegues cutáneos mediante plicómetro, la ecografía, la TC y la RM son técnicas de diagnóstico por imágenes que pueden usarse para diagnosticar la esteatosis hepática. ${ }^{13,18,26-28}$ De estas modalidades, la ecografía es un método asequible, ampliamente disponible y sin radiación. ${ }^{26}$ Se demostró que la ecografía hepática también puede usarse para cuantificar la esteatosis en los pacientes pediátricos, y la evaluación ecográfica tiene una correlación sólida con el grado de esteatosis en la biopsia de hígado. ${ }^{28,29}$ La desventaja de la ecografía radica en que si el hígado tiene menos de un $30 \%$ de grasa, la sensibilidad disminuye. ${ }^{5,13}$ Si bien hay algunos informes sobre el uso de modalidades de diagnóstico por imágenes como la TC y la RM para diagnosticar la esteatosis hepática, no se las suele considerar viables en niños debido a su costo, la posible necesidad de sedación, su disponibilidad relativamente limitada y la radiación propia de la TC. ${ }^{5,30}$ En nuestro estudio, se utilizó la ecografía para evaluar el parénquima hepático y detectar esteatosis, y se observó que todos los pacientes tenían cierto grado de 
esteatosis hepática, aunque casi tres cuartos tenían grado 1 .

El aumento de la adiposidad corporal observado en la obesidad se ha relacionado con trastornos metabólicos, y se ha intentado determinar la cantidad de grasa corporal mediante diferentes parámetros antropométricos. Otros estudios se han centrado en las asociaciones entre la cantidad de tejido adiposo visceral y subcutáneo y la esteatosis hepática y la dislipidemia. ${ }^{31-35}$ Se ha informado de forma consistente que una mayor grasa visceral está asociada a la desregulación metabólica y a una concentración elevada de enzimas hepáticas. ${ }^{32,33}$ Yamaguci y cols. hallaron una fuerte correlación entre el tejido adiposo visceral y el perfil lipídico sérico y las transaminasas en niños obesos. ${ }^{33}$ Bibiloni y cols., demostraron que el IMC y la obesidad abdominal se asocian a la prevalencia de, al menos, un marcador de concentración anormal de lípidos, y el C-LDL bajo fue la dislipidemia más frecuente entre sus pacientes. ${ }^{34}$ Bijari y cols., observaron una relación entre la obesidad central y cambios adversos en el perfil lipídico y recomendaron examinar a los pacientes para detectar obesidad central a fin de prevenir enfermedades cardiovasculares. ${ }^{35}$ Asayama y cols., investigaron la relación entre el espesor de la grasa visceral o la razón entre la grasa visceral y subcutánea y varios parámetros antropométricos e informaron que el tejido adiposo visceral tenía una mejor correlación con los valores de lípidos séricos que representaban un riesgo metabólico para los niños. ${ }^{32}$ Todos estos estudios se centraron en evaluar la obesidad central y visceral, que requiere el uso de técnicas de imagen transversal como la TC o la RM.

No obstante, hay pocos estudios donde se investigó la relación entre la cantidad de grasa subcutánea y los factores de riesgo metabólico. ${ }^{36,37}$ Mager y cols., mostraron en su estudio que la grasa subcutánea del tronco, considerada como la suma de la grasa subcutánea de las regiones abdominal, suprailíaca y subescapular, predice valores bioquímicos hepáticos anormales y dislipidemia. ${ }^{37}$ Lee y cols., utilizaron el espesor de la GSA como único parámetro para reflejar la cantidad de grasa subcutánea y observaron una correlación positiva entre el espesor de la GSA y el IMC y la esteatosis hepática. ${ }^{31}$ Nuestros resultados también mostraron que el espesor de la GSA de la línea media incrementaba un $64,8 \%$ en la esteatosis de grado 1 y un $55,8 \%$ en la esteatosis de grado 2 por cada $1 \mathrm{~kg} / \mathrm{m}^{2}$ de aumento en el IMC, lo que sugiere que un aumento en el IMC hace que la grasa se deposite no solo en el hígado sino también en los tejidos subcutáneos. También se observó una correlación positiva entre el espesor de la GSA y el IMC. Lee y cols., hallaron una correlación entre el espesor de la GSA y las concentraciones de CT, C-LDL y ALT. ${ }^{31}$ De manera similar, en nuestros resultados, un aumento en la concentración de TG y C-LDL en los pacientes con esteatosis de grado 3 se asoció a un valor más elevado en el espesor de la GSA de la línea media. Además, en nuestro estudio, un aumento en las concentraciones de TG y AST se asoció a valores más elevados en el espesor de la GSA de los flancos en la esteatosis de grado 3. También se demostró que los valores de ALT de los pacientes con esteatosis de grado 1 eran significativamente menores que los de aquellos que tenían esteatosis de grados 2 y 3 . Estos resultados sugieren que un aumento en el espesor de la GSA se asoció a un mayor trastorno del metabolismo lipídico, grados más elevados de esteatosis y concentraciones más altas de AST. De este modo, la medición ecográfica del espesor de la GSA puede incluirse en los estudios diagnósticos habituales de los niños obesos. Probablemente esto permita identificar y tratar de forma más temprana a los niños con un mayor riesgo de dislipidemia y compromiso hepático $\mathrm{y}$, de esta forma, prevenir las complicaciones relacionadas con la obesidad que puedan surgir más adelante. Una vez que se implementan cambios en el estilo de vida y una dieta adecuada, el espesor de la GSA también puede usarse como un método fácil para controlar a los niños durante este período, a medida que los parámetros de riesgo relacionados con la obesidad regresan a valores normales.

Este estudio presenta ciertas limitaciones. En primer lugar, la evaluación del esteatosis hepática mediante ecografía depende del operador, es subjetiva y, por ende, cualitativa. Además, si la esteatosis afecta menos del $30 \%$ del parénquima hepático, la sensibilidad y la especificidad disminuyen. ${ }^{13,30}$ Por lo tanto, es probable que sea menos reproducible y no ofrezca datos cuantitativos sobre la esteatosis, si bien un estudio a gran escala realizado por Schlecht y cols., informó que la ecografía tenía un alto nivel de reproducibilidad y validez para medir el espesor del tejido subcutáneo, particularmente en niños no obesos. ${ }^{15}$ Por lo tanto, la evaluación cuantitativa de la esteatosis hepática mediante RM en estudios futuros 
podría esclarecer mejor este asunto y ayudar a confirmar nuestros resultados de una manera más confiable. En segundo lugar, en nuestro estudio no se realizó el análisis anatomopatológico del esteatosis hepática mediante una biopsia de hígado; esto otorgaría una mayor fiabilidad a nuestros resultados y también aclararía otros posibles motivos incluidos en el diagnóstico diferencial. ${ }^{13}$ Además, no se recopilaron datos sobre el origen étnico ni la circunferencia de cintura de los pacientes, los cuales deberían tenerse en cuenta al evaluar los resultados. Otra limitación fue que la cantidad de pacientes con esteatosis de grado 3 fue pequeña en comparación con los otros grupos, lo que disminuye en gran medida las certezas relativas a la interpretación de los resultados de este subgrupo. Por lo tanto, consideramos que es necesario realizar estudios adicionales que incluyan una mayor cantidad de pacientes con esteatosis hepática de grado 3 para sacar conclusiones más confiables.

\section{CONCLUSIONES}

En conclusión, tanto el espesor de la GSA de la línea media como el de los flancos tuvieron una correlación positiva con los TG en los niños obesos con esteatosis hepática de grado 3; mientras que, en los casos de esteatosis de grado 1 , el espesor de la GSA de la línea media tuvo una correlación positiva con el IMC, el CT, el C-LDL y la AST. No obstante, estas asociaciones deben confirmarse mediante estudios futuros a gran escala, quizá con modalidades como la RM, que permitan evaluar la esteatosis hepática de forma cuantitativa.

\section{REFERENCIAS}

1. Güngör NK. Overweight and obesity in children and adolescents. J Clin Res Pediatr Endocrinol. 2014; 6(3):129-43.

2. Han JC, Lawlor D, Kimm SYS. Childhood Obesity-2010: Progress and Challenges. Lancet. 2010; 375(9727):1737-48.

3. Lobstein T, Baur L, Uauy R, IASO International Obesity TaskForce. Obesity in children and young people: a crisis in public health. Obes Rev. 2004; 5(Suppl 1):4- 104.

4. Nicolai JP, Lupiani JH, Wolf A J. An Integrative approach to obesity. En: Rakel D (ed). Integrative Medicine. 3rd ed. Philadelphia, PA: Saunders-Elsevier; 2012.P.364-75.

5. Feldstein AE, Patton-Ku D, Boutelle KN. Obesity, Nutrition and Liver Disease in Children. Clin Liver Dis. 2014; 18(1):21931.

6. Freedman DS, Khan LK, Serdula MK, Dietz WH, et al. The relation of childhood BMI to adult adiposity: the Bogalusa Heart Study. Pediatrics. 2005; 115(1):22-7.

7. Pulgaron ER, Delamater AM. Obesity and type 2 diabetes in children: epidemiology and treatment. Curr Diab Rep. 2014; 14(8):508.

8. Juonala M, Magnussen CG, Berenson GS, Venn A, et al. Childhood adiposity, adult adiposity, and cardiovascular risk factors. N Engl J Med. 2011; 365(20):1876-85.

9. Nobili V, Reale A, Alisi A, Morino G, et al. Elevated serum ALT in children presenting to the emergency unit: Relationship with NAFLD. Dig Liver Dis. 2009; 41(10):74952.

10. Parekh S, Anania FA. Abnormal lipid and glucose metabolism in obesity: implications for nonalcoholic fatty liver disease. Gastroenterology. 2007; 132(6):2191-207.

11. Donnelly KL, Smith CI, Schwarzenberg SJ, Jessurun J, et al. Sources of fatty acids stored in liver and secreted via lipoproteins in patients with nonalcoholic fatty liver disease. J Clin Invest. 2005; 115(5):1343-51.

12. Ko JS, Yoon JM, Yang HR, Myung JK, et al. Clinical and histological features of nonalcoholic fatty liver disease in children. Dig Dis Sci. 2009; 54(10):2225-30.

13. Vajro P, Lenta S, Socha P, Dhawan A, et al. Diagnosis of nonalcoholic fatty liver disease in children and adolescents: position paper of the ESPGHAN Hepatology Committee. J Pediatr Gastroenterol Nutr. 2012; 54(5):700-13.

14. SemizS,OzgörenE,SabirN.Comparisonofultrasonographic and anthropometric methods to assess body fat in childhood obesity. Int J Obes (Lond). 2007; 31(1):53-8.

15. Schlecht I, Wiggermann P, Behrens G, Fischer B, et al. Reproducibility and validity of ultrasound for the measurement of visceral and subcutaneous adipose tissues. Metabolism. 2014; 63(12):1512-9.

16. Wagner DR. Ultrasound as a tool to assess body fat. J Obes. 2013; 2013:280713.

17. Whitlock EP, Williams SB, Gold R, Smith PR, Shipman SA. Screening and interventions for childhood overweight: a summary of evidence for the US Preventive Services Task Force. Pediatrics. 2010; 116(1):e125-44.

18. Dasarathy S, Dasarathy J, Khiyami A, Joseph R, et al. Validity of real time ultrasound in the diagnosis of hepatic steatosis: a prospective study. J Hepatol. 2009; 51(6):1061-7.

19. Pulgarón ER. Childhood obesity: A review of increased risk for physical and psychological comorbidities. Clin Ther. 2013; 35(1):A18-32.

20. Burt Solorzano CM, McCartney CR. Obesity and the pubertal transition in girls and boys. Reproduction. 2010; 140(3):399-410.

21. Schwimmer JB, Deutsch R, Kahen T, Lavine JE, et al. Prevalence of fatty liver in children and adolescents. Pediatrics. 2006; 118(4):1388-93.

22. Jung JH, Jung MK, Kim KE, Kwon AR, et al. Ultrasound measurement of pediatric visceral fat thickness: correlations with metabolic and liver profiles. Ann Pediatr Endocrinol Metab. 2016; 21(2):75-80.

23. SundaramSS, Zeitler $P$, Nadeau K. The metabolic syndrome and nonalcoholic fatty liver disease in children. Curr Opin Pediatr. 2009; 21(4):529-35.

24. Marzuillo P, Miraglia del Giudice E, Santoro N. Pediatric fatty liver disease: Role of ethnicity and genetics. World J Gastrenterol. 2014; 20(23):7347-55.

25. Staiano A, Katzmarzyk P. Ethnic and sex differences in body fat, visceral, and subcutaneous adiposity in children and adolescents. Int J Obes (Lond). 2012; 36(10):1261-9.

26. Sabir N, Sermez Y, Kazil S, Zencir M. Correlation of abdominal fat accumulation and liver steatosis: importance of ultrasonographic and anthropometric measurements. Eur J Ultrasound. 2001; 14(2-3):121-8.

27. Mazhar SM, Shiehmorteza M, Sirlin CB. Noninvasive assessment of hepatic steatosis. Clin Gastrenterol Hepatol. 2009; 7(2):135-40.

28. Shannon A, Alkhouri N, Carter-Kent C, Monti L, et al. Ultrasonographic quantitative estimation of hepatic steatosis in children with NAFLD. J Pediatr Gastroenterol Nutr. 2011; 53(2):190-5.

29. Eifler RV. The role of ultrasonography in the measurement of subcutaneous and visceral fat and its correlation with 
hepatic steatosis. Radiol Bras. 2013; 46(5):273-8.

30. Vos M, Abrams S, Barlow S, Caprio S, et al. NASPGHAN clinical practice guideline for the diagnosis and treatment of Nonalcoholic Fatty Liver Disease in children: recommendations from the expert Committee on NAFLD (ECON) and the North American Society of Pediatric Gastroenterology, Hepatology and Nutrition (NASPGHAN). J Pediatr Gastroenterol Nutr. 2017; 64(2):31934.

31. Lee SH, Kim D, Baek MY, Tchah H, et al. Abdominal Subcutaneous Fat Thickness Measured by Ultrasonography Correlates with Hyperlipidemia and Steatohepatitis in Obese Children. Pediatr Gastroenterol Hepatol Nutr. 2015; 18(2):108-14.

32. Asayama K, Dobashi K, Hayashibe H, Kodera K, et al. Threshold values of visceral fat measures and their anthropometric alternatives for metabolic derangement in Japanese obese boys. Int J Obes Relat Metab Disord. 2002; 26(2):208-13.
33. Yamaguchi J, Oguni T, Konishi K, Mino M. Abdominal adiposity with respect to the proportion of intra-abdominal visceral fat to extra-abdominal fat (VS ratio) in Japanese childhood obesity. Pathophysiol. 1996; 3(1):29-35.

34. Bibiloni MD, Salas R, De la Garza YE, Villarreal JZ, et al. Serum lipid profile, prevalence of dyslipidaemia, and associated risk factors among Northern Mexican adolescents. J Pediatr Gastroenterol Nutr. 2016; 63(5):544-9.

35. Bijari B, Taheri F, Chahkandi T, Kazemi T, et al. The relationship between serum lipids and obesity among elementary school in Birjand: a case control study. J Res Health Sci. 2015; 15(2):83-7.

36. Deeb A, Attia S, Mahmoud S, Elhaj G, Elfatih A. Dyslipidemia and fatty liver disease in overweight and obese children. J Obes. 2018; 2018:8626818.

37. Mager DR, Yap J, Rodriguez-Dimitrescu C, Mazurak V, et al. Anthropometric Measures of visceral and subcutaneous fat are important in the determination of metabolic dysregulation in boys and girls at risk for nonalcoholic fatty liver disease. Nutr Clin Pract. 2013; 28(1):101-11.

\section{Archivos hace 75 años}

Faculted de Ciencies Medices de Bvenes Aires. Instirufo de Pediatria y Purricultura Proferor: Dr, Juan P. Garraten

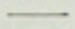

ENFISEMA MEDIASTINICO Y SUBCUTANEO EN EL CURSO DE UNA CRISIS ASMATICA. OBSERVACION CLINICA *

$$
\text { por Los }
$$

DRES. ALFREDO E. LARGUIA y ERNESTO T. SOJO

Una noción bòen difundida es que la mayoría de las observaciones de enfisema subcutáneo, originado a partir de las cavidades pleurales, han tenido su origen en traumas del tórax, en maniobras intempestivas de la cirugía torácica, en traqueotomías, punciones, etc., o bìen son consecutivos a la colapeoterapia del pulmón tuberculoso y en especial al neumotórax valvular hipertensivo, también de origen baciloso.

Los casos de enfisema subcutáneo y mediastínico de origen no tuberculoso ni traumático o accidental, no son de obnervación corriente. Recaen casi siempre en nî́os o sujetos jóvenes y han sido descriptos en cl curso de neumonias ", bronconeumonias " ${ }^{2}$, gripe, sarampión *, coque. luche, difterias malignas ", consecutivos a fiebres tíficas", como consecuencia de la introducción de cuerpos extraños en las vías aéreas, etc.

La aparición del enfisema subcutáneo y mediastínico, como sintoma o complicación de crisis de asma bronquial, es de observación mucho menos frecuente aún, y ha sido scñalada sólo en contadas ocasiones.

Entre nosotros, Schweizer y Senct ${ }^{\mathrm{r}}$, en 1937 , presentaron a la Sociedad Argentina de Pediatría una observación en una niña de 3 años, y muy recientemente Fongi y Roepide ${ }^{*}$ en una revisión de conjunto sobre el tema han podido reunir sólo 31 casos en la literatura mundial. a los cuales agregan ellos 2 nuevas ohservaciones en sujetos de 22 , 26 años.

Este número relativamente bajo de observaciones, nos ha hecho creer que pueda tener interés el relato de un nuevo caso, que de sintomatologia inicial espectacular, tuvo afortunadamente una evolución favorable.

Ia historia clinica resumida de nuetro enfermo es la siguiente:

El texto completo se encuentra disponible en la versión electrónica de este número. 